\title{
Pathogenicity and Host Susceptibility of Penicillium spp. on Citrus
}

Johannes Petrus Louw and Lise Korsten, University of Pretoria, Department of Microbiology and Plant Pathology, New Agricultural Building, Lunnon Road, Hillcrest, 0083, South Africa

\begin{abstract}
Louw, J. P., and Korsten, L. 2015. Pathogenicity and host susceptibility of Penicillium spp. on citrus. Plant Dis. 99:21-30.

Citrus fruit are exposed to numerous postharvest pathogens throughout the fresh produce supply chain. Well-known postharvest citrus fruit pathogens are Penicillium digitatum and $P$. italicum. Lesser-known pathogens include $P$. crustosum and P. expansum. This study examined pathogenicity and aggressiveness of Penicillium spp. present in fresh fruit supply chains on various Citrus spp. and cultivars. The impact of different inoculation methods and storage conditions on decay were also assessed. $P$. digitatum and $P$. italicum were the most aggressive Penicillium spp. on citrus but aggressiveness varied significantly over the evaluated citrus range. Decay and tissue-response lesions caused by

P. crustosum were observed on 'Nules Clementine', 'Nova', 'Owari Satsuma', 'Delta Valencia', 'Cambria Navel', 'Eureka' seeded, and 'Star Ruby' for the first time. Likewise, these lesions caused by $P$. expansum were noted on Nules Clementine, Owari Satsuma, Delta Valencia, 'Midknight Valencia', and Eureka seeded for the first time. Tissue-response lesions affect fruit quality and some Penicillium spp. sporulated from the lesions, causing the inoculated species to complete their life cycle. New citrus-Penicillium spp. interactions were observed and the importance of monitoring inoculum loads of pathogens and nonhost pathogens were highlighted.
\end{abstract}

Citrus has an economic and nutritional importance worldwide (15). South Africa exported 64\% of its citrus produced in 2011 as fresh fruit, earning a gross export total of over $\$ 817$ million (5). Disease caused by fungal pathogens contribute greatly to postharvest losses $(6,18)$. The most important postharvest pathogens of citrus, Penicillium digitatum (Pers.) Sacc., can account for $90 \%$ of total losses $(6,18)$. Additional Penicillium spp. of concern in the citrus industry are $P$. italicum Wehmer and $P$. ulaiense H.M. Hsieh, H.J. Su \& Tzean $(4,18,19,21,22)$. Previous reports also indicate $P$. crustosum Thom $(3,10), P$. expansum Link (34), and $P$. fellutanum Biourge (24) as citrus pathogens; however, information associating these species with losses in citrus industries is lacking. Data on $P$. fellutanum have not been confirmed.

Little is known about the pathogenicity of P. crustosum on citrus. Garcha and Singh (10) reported total decay of mandarin fruit inoculated with $P$. crustosum after 8 days. The cultivar, inoculum concentration, and incubation temperature were not specified. The described symptoms included watery spots which later extend deeper into the tissue, white mycelial tufts that turn bluish-green when sporulating, and a fermented odor. Arrebolla et al. (3) confirmed $P$. crustosum as pathogenic on citrus by reproducing lesions $(17.25 \pm 5.2 \mathrm{~mm}$ in diameter; incidence $=36 \%)$ on 'Valencia' orange fruit kept in modified atmosphere packaging at $25^{\circ} \mathrm{C}$ for 12 days. Unfortunately, the research focus did not further address the P. crustosum-citrus interaction and was mainly focused on control.

Macarisin et al. (17) reported $P$. expansum infections on citrus (lemon, grapefruit, and orange) and attributed the arrested infection to the host's production of reactive oxygen species. Vilanova et al. (34) were able to facilitate development of $P$. expansum decay on commercially mature and over-mature orange fruit ('Navelina' and Valencia) with high inoculum concentrations $\left(10^{6}\right.$ and $10^{7}$ conid$\mathrm{ia} / \mathrm{ml})$. Lesions averaged 3 and $8 \mathrm{~mm}$ in diameter on Valencia fruit inoculated with $10^{6}$ and $10^{7}$ conidia/ml, respectively. The Valencia orange fruit were incubated for 17 days at $20^{\circ} \mathrm{C}$ and $85 \%$ relative humidity $(\mathrm{RH})$. Lesions on Navelina were larger, averaging from 10 to $35 \mathrm{~mm}$ after 11 days for the highest inoculum concentration.

Corresponding author: L. Korsten, E-mail: lise.korsten@up.ac.za

Accepted for publication 19 June 2014.

http://dx.doi.org/10.1094/PDIS-02-14-0122-RE

(C) 2015 The American Phytopathological Society
Penicillium spp. are ubiquitous organisms, commonly found in air, water, soil, indoors, and in numerous fresh and processed food products $(8,21)$. These characteristics and intricate fruit trade networks contribute to the dissemination of the fungus, often resulting in high inoculum build-up in the diverse environments of the fruit handling and marketing chain. Fruit trade networks can result in various fruit types originating from different countries being handled, transported, and stored together (31). These fruit can harbor different pathogens which can lead to cross-contamination when handled together. This exposes citrus fruit to typical postharvest pome fruit pathogens (i.e., $P$. crustosum and $P$. expansum) and pome fruit to the typical postharvest citrus pathogens (i.e., $P$. digitatum and P. italicum). Recently, Louw and Korsten (16) reported $P$. digitatum pathogenic on pome fruit, and Vilanova et al. (34) reported decay of orange fruit caused by $P$. expansum.

The aim of this study was to provide better understanding of the infective potential of Penicillium spp. in the citrus supply chain, and confirm the pathogenicity ("the capability to cause disease"; 1) and aggressiveness ("the quantitative component of pathogenicity"; 20) of different Penicillium spp. on various Citrus spp. and cultivars. The significance of different inoculation methods and storage conditions on decay development was also assessed.

\section{Materials and Methods}

Fungal cultures. The isolates of $P$. digitatum, $P$. italicum, $P$. crustosum, and $P$. expansum used in this study are listed in Table 1. With the exception of $P$. solitum that was replaced by $P$. italicum, they are the same isolates used by Louw and Korsten (16). Criteria for selecting the isolates and species were based on their presence in export chains (pome and citrus) and pathogenic potential on citrus. Cultures were single-spored, grown on malt extract agar (MEA) (Merck, Biolab Diagnostics [Pty.] Ltd., Johannesburg, South Africa) and incubated in darkness at $25^{\circ} \mathrm{C}$ for 3 weeks prior to fruit inoculation studies. The species were consistently used throughout the study.

Fruit origin and handling. Commercially harvested and graded citrus fruit were obtained for the trials from commercial export farms in the Eastern Cape Province. Mandarin cultivars were 'Nules Clementine' (Citrus clementina hort. ex Tanaka), 'Nova' (hybrid: $C$. clementina $\times$ 'Orlando' tangelo [C. paradisi Macf. $\times C$. tangerina hort. ex Tanaka]), and 'Owari Satsuma' (C. unshiu Marcow.). Sweet orange (C. sinensis L. Osbeck) cultivars were 'Navel' ('New Hall', 'Palmer', and 'Cambria') and Valencia ('Midknight' and 'Delta'). The lemon and grapefruit cultivars were 
'Eureka' seeded (C. limon (L.) Burm. f.) and 'Star Ruby' (C. paradisi Macf.), respectively.

Fruit were inoculated at two different laboratories depending on the seasonal availability of fruit. The first inoculations took place in a laboratory at the citrus packinghouse in the Eastern Cape and the second was done at the University of Pretoria (UP) facilities. Fruit inoculated at the packinghouse were Nules Clementine, Nova, Owari Satsuma, 'New Hall Navel', 'Palmer Navel', and Eureka seeded. They were delivered directly after handpicking. Fruit were inoculated 1,2, or 3 days after delivery, depending on availability and seasonality. Owari Satsuma was the only cultivar that was stored ( 9 days at $\pm 4^{\circ} \mathrm{C}$ ) prior to inoculation. Fruit inoculated at UP were 'Cambria Navel', 'Midknight Valencia', 'Delta Valencia', Eureka seeded, and Star Ruby. The fruit were cargo shipped from Port Elizabeth to Johannesburg (transported under cargo holding conditions and cold stored on arrival), collected within $24 \mathrm{~h}$, and transported from Johannesburg to Pretoria, where trials started a day thereafter. Fruit used in trials were physiologically mature according to the natural quality standards for export (26), without any postharvest treatment.

Confirming Penicillium spp. pathogenicity on citrus. An initial pathogenicity trial was conducted to determine the pathogenicity of major postharvest Penicillium spp. pathogens encountered in fruit storage and handling chains (16) on citrus. Each isolate from the citrus chain (Table 1) was inoculated into fruit of mandarin (Nules Clementine), sweet orange (New Hall Navel), lemon (Eureka seeded), and grapefruit (Star Ruby). Fruit were surface sterilized prior to inoculation by dipping in $0.002 \%$ sodium hypochlorite solution for more than 5 min and allowed to air dry on a surface-sterilized table overlaid with paper towels. A set of 10 fruit were wounded ( 1.5 by 1.5 by $2 \mathrm{~mm}$ ) on opposite sides by piercing the pericarp prior to inoculation. Each inoculated fruit represented a replicate. A metal wire protruding from a cork was used for wounding to ensure that wounds were uniform. Wounds were inoculated with conidial suspensions at $6.3 \times 10^{4}$ conidia $/ \mathrm{ml}$. Noninoculated fruit were included as controls. Conidial suspensions were prepared in sterile Ringers solutions (physiological saline solution, Merck) and $0.05 \%$ Tween 80 (Associated Chemical Enterprises, Johannesburg). Concentrations were determined using a hemocytometer. A $10-\mu$ l conidial suspension was deposited with a micropipette into each wound. A strip of Paraflim was taped around the fruit, covering the wounds to avert cross-contamination. Fruit were arranged in a completely randomized design (CRD) on a disinfected table and incubated for 7 days under ambient conditions $\left(20\right.$ to $\left.22^{\circ} \mathrm{C}\right)$. Lesions were measured on the third, fifth, and seventh day postinoculation. Horizontal and vertical (calyx axis vertical) measurements were taken. The means of control wounds were subtracted from the diameter of decay and tissue-response lesions. Tissue-response lesions were lesions caused by hypersensitive response (HR) reactions (infection was arrested after reaching a certain size). The experiment was repeated.

A comparative pathogenicity trial was conducted using Penicillium spp. environmental isolates from both fruit supply chains (Table 1). The aim of the trial was to determine whether a citrus environment harbors isolates similar to those from a pear supply chain environment with respect to pathogenicity and aggressiveness. The methodology used was similar to the initial Penicillium spp. trial. Five mandarin fruit ('Nules Clementine') were inoculated with each Penicillium spp. isolate. Five wounded but noninoculated fruit served as controls. Fruit were arranged in a CRD and the experiment was repeated. Incubation and data recording was as previously described.

Comparison of inoculation methods. Three methods were compared to select the most suited one for the citrus fruit inoculation trials. Methods include inoculation via conidial suspensions, plugs (MEA), or aerially dispersed conidia. Only isolates from the citrus environment were used in this and following trials (Table 1). Ten Eureka seeded lemon fruit (each a replicate) were wounded and inoculated with each Penicillium sp. for each method. Wounding of fruit for inoculation using conidial suspensions were as previously described but wounding for plug inoculations and air inoculations were made with a 5-mm-diameter sterile cork borer, 2 to 3 $\mathrm{mm}$ deep. Plugs ( $5 \mathrm{~mm}$ in diameter), intended for inoculation via plugs were cut from 2- to 3-week-old cultures (MEA) and placed into the wound sites with a sterilized scalpel. Control fruit were wounded but received no Penicillium agar plugs. Parafilming and incubation of fruit and data recording were as described earlier.

Air inoculation of Eureka seeded lemon fruit were conducted inside an inoculation chamber (Fig. 1) assembled in a biosafety cabinet $(14,23)$. The chamber was composed of a metal frame overlaid with a plastic bag $(61$ by $102.5 \mathrm{~cm})$. The inside was sterilized with $70 \%$ ethanol and allowed to air dry. Ten wounded fruit (as described earlier) were placed in the chamber with a 2- to 3-week-old sporulating Penicillium culture (MEA) plate. An open sterile MEA plate $(65 \mathrm{~mm})$ was positioned among the fruit in the chamber to confirm conidial dissemination. A disinfected fan (YJ 58-12C motor with a $15 \mathrm{~cm}$-diameter double-blade prop) was also placed inside, behind the open Penicillium plate. The chamber was closed to ensure air circulation across the plate and prevent air entering from outside while the fan was operational $(10 \mathrm{~min})$. The fruit were removed, wounds were covered with a strip of Parafilm, fruit were incubated under ambient conditions, and recording of data was as described in the pathogenicity trial. Control fruit were as described with plug inoculation. Fruit were arranged according to a complete randomized block design (CRBD) with a factorial arrangement. The trial was repeated. The size of the lesions was adjusted by subtracting the mean diameter of the wound in control treatments from the mean of measured lesions.

Penicillium decay of lemon fruit under cold storage conditions. The effect of cold storage conditions on decay development caused by Penicillium spp. was evaluated on Eureka seeded lemon fruit. Two sets of 10 surface-sterilized fruit were inoculated with each Penicillium sp. via conidial suspensions. Each inoculated fruit counted as a replicate. Wounds were covered with Parafilm as described earlier. One set of fruit was incubated under ambient conditions for 7 days $\left(21.9 \pm 0.4^{\circ} \mathrm{C}\right.$ and $\left.43.6 \pm 4.6 \% \mathrm{RH}\right)$ and another under refrigerated conditions for 26 days $\left(5.0 \pm 0.6^{\circ} \mathrm{C}\right.$ and $86.4 \pm$ $4.4 \% \mathrm{RH})$. Lesion diameters for ambient incubated fruit were measured as described earlier. Measurements for fruit stored in the cold room started on the 1st day of lesion development (observation) and

Table 1. Penicillium citrus and pear chain environment isolates used in this study

\begin{tabular}{|c|c|c|c|c|c|}
\hline Isolate code & Species & Chain & Date & $\begin{array}{l}\text { Country } \\
\text { of origin }\end{array}$ & Source (isolate located) \\
\hline $\mathrm{PdC}$ & Penicillium digitatum & Citrus & 2009-2010 & The Netherlands & $\begin{array}{l}\text { Floor of distributer/repack facility; reintroduced into plums and } \\
\text { isolated from lesions (2011) }\end{array}$ \\
\hline $\mathrm{PdP}$ & P. digitatum & Pear & 2011 & United Kingdom & Small waste bin of repack facility \\
\hline $\mathrm{PiC}$ & P. italicum & Citrus & 2009-2010 & Germany & Air of distributer/repack facility/cold room \\
\hline PiP & P. italicum & Pear & 2011 & United Kingdom & Air of repack facility receive area \\
\hline $\mathrm{PcC}$ & P. crustosum & Citrus & 2009-2010 & Germany & Air, walls or floor of packhouse \\
\hline PcP & P. crustosum & Pear & 2011 & South Africa & Wall of packhouse holding area \\
\hline $\mathrm{PeC}$ & P. expansum & Citrus & 2009-2010 & Germany & $\begin{array}{l}\text { Wall of distributer/repack facility; reintroduced into apples and } \\
\text { isolated from lesions (2011) }\end{array}$ \\
\hline $\mathrm{PeP}$ & P. expansum & Pear & 2011 & United Kingdom & Air of cold storage facility \\
\hline
\end{tabular}


continued every 2nd day thereafter up to the 26th day. Means of control wounds were also calculated to subtract from means of measured lesions. The experiment was repeated and arranged in a CRBD. Wounded but noninoculated fruit served as controls.

Penicillium spp. aggressiveness on citrus. The aggressiveness of each Penicillium sp. was assessed on mandarin (Nules Clementine, Nova, and Owari Satsuma), sweet orange (New Hall Navel, Palmer Navel, Cambria Navel, Midknight Valencia, and Delta Valencia), lemon (Eureka seeded), and grapefruit (Star Ruby) fruit. Ten surface-sterilized fruit from each cultivar was inoculated with each Penicillium sp. via conidial suspensions. Each inoculated fruit represented a replicate. Fruit were randomized (factorial arrangement on a CRD) and the experiment was repeated. The incubation of fruit and data collection was as previously described.

Reisolation from fruit, identification, and preservation. Isolations were made from two fruit from each Penicillium sp.-cultivar interaction from each experiment in every trial. Variables were also involved in the case of the isolate comparison, inoculation method, and cold room trials: different isolates, inoculation methods, or incubation condition. In these cases, two isolates were also made for each Penicillium sp.-cultivar interaction for each variable from both experiments in a trial. Isolates (MEA) were incubated as previously described. Sufficient growth from pure cultures revealed visual similarities among cultured isolates. One culture from each cultivar-Penicillium sp. interaction and variable was identified and preserved (two water- and two cryo-preservations per culture), as described by Louw and Korsten (16). The Penicillium $\beta$-tubulin gene was amplified in a CFX Connect Real-Time PCR Detection System (Bio-Rad, Singapore) using the Bt2a and Bt2b primers (11) and EvaGreen dye (Biotium Inc., Hayward, USA). The PCR cycles were $95^{\circ} \mathrm{C}$ for $3 \mathrm{~min} ; 35$ cycles of $94^{\circ} \mathrm{C}$ for $30 \mathrm{~s}$ followed by $57^{\circ} \mathrm{C}$ for $45 \mathrm{~s}$ and $72^{\circ} \mathrm{C}$ for $2 \mathrm{~min}$; and a final elongation at $72^{\circ} \mathrm{C}$ for 7 min. Isolates were grouped via polymerase chain reaction restriction fragment length polymorphism (PCR-RFLP) and identified via sequencing. Sequencing confirmed the identity of allocated PCR-RFLP groupings. PCR-RFLP was performed by restriction digestion of $\beta$-tubulin genes with BfaI (isochitzomer; FspBI) (Inqaba, Pretoria, South Africa) and observing fragments separated on $3 \%$ agarose gels ( $75 \mathrm{~V}$ over 3 to $5 \mathrm{~h}$ ) with a 100-bp ladder/marker. Sequencing PCR was conducted in an Eppendorf Masterycler Pro $\mathrm{S}$ (Hamburg, Germany) thermocycler. Cycles were $95^{\circ} \mathrm{C}$ for $1 \mathrm{~min}$ followed by 25 cycles of $96^{\circ} \mathrm{C}$ for $10 \mathrm{~s}, 50^{\circ} \mathrm{C}$ for $5 \mathrm{~s}$, and $60^{\circ} \mathrm{C}$ for 4 min. Sequences were analyzed in an ABI 3500 Genetic Analyzer (Applied Biosystems, Foster City, USA).

Statistical analysis. SAS software (version 9.2; SAS Institute Inc., Carry, NC) was used for statistical analysis of data. Each inoculated fruit accounted for a replicate. The two wounds on each fruit provided four subsamples (two wounds, each with a horizontal and vertical diameter). Respective means of control wounds were subtracted from lesions prior to statistical analysis. When the Bartlett's test for homogeneity revealed similarity between experiments in trials, experiments were pooled. Means were separated using Fisher protected least significant difference.

Disease incidence (percent) and lesion diameters (in millimeters) from the pathogenicity trial were recorded to calculate disease intensity. Disease intensity combines disease incidence with disease severity to express disease concern linked to each Penicillium sp. on a specific crop. Tissue-response lesions, although they are not typical fruit decay lesions and infection is arrested, were considered because they have an important impact on fruit quality and, in some cases, became significantly large. Only lesions significantly larger than the mean of the control wound diameters were considered for calculating disease intensity. Disease intensity was previously described and used by Van Eeden and Korsten (30) and Louw and Korsten (16). Disease intensity $=\left[(d \times F) /\left(D \times T_{\mathrm{n}}\right)\right] \times$ 100 , where $d=$ degree of disease severity assessed (mean lesion diameter), $F=$ frequency (number of lesions), $D=$ maximum lesion diameter measurable, and $T_{\mathrm{n}}=$ total number of fruit (in our case, lesions) examined.

\section{Results}

Confirming Penicillium spp. pathogenicity on citrus. No significant difference was observed between the independent pathogenicity experiments $(P=0.4)$. Only $P$. digitatum and $P$. italicum caused large lesions on mandarin (Nules Clementine), sweet orange (New Hall Navel), lemon (Eureka seeded), and grapefruit (Star Ruby). Tissue-response lesions included in disease intensity were caused by $P$. crustosum and $P$. expansum on Nules Clementine and Star Ruby (Table 2). Additional tissue-response lesions caused by $P$. expansum and $P$. crustosum considered worth mentioning were observed on Nules Clementine, $P$. crustosum $=7.2 \pm$ $2.3 \mathrm{~mm}$ lesion diameter (ld) (22.2\%); New Hall Navel, P. crustosum $=8.1 \mathrm{~mm} \mathrm{ld}(5.9 \%)$, and $P$. expansum $=4.9 \pm 1.0 \mathrm{~mm} l d$ (11.1\%); Star Ruby, P. crustosum $=6.7 \pm 2.3 \mathrm{~mm} l d(27.8 \%)$ and $P$. expansum $=5.0 \pm 3.8 \mathrm{~mm}$ ld $(15.0 \%)$; and Eureka seeded, $P$. crustosum $=4.3 \pm 2.9 \mathrm{~mm} l d(10.5 \%)$.

Independent experiments comparing citrus- and pear-chain environment isolates on mandarin fruit were not significantly different $(P=0.8)$. Importantly, significant differences were not found when Penicillium spp. lesion sizes of pear and citrus isolates were compared. Three t-groupings formed; $P$. digitatum isolates (group a), $P$. italicum isolates (group b), and the remainder of the Penicillium

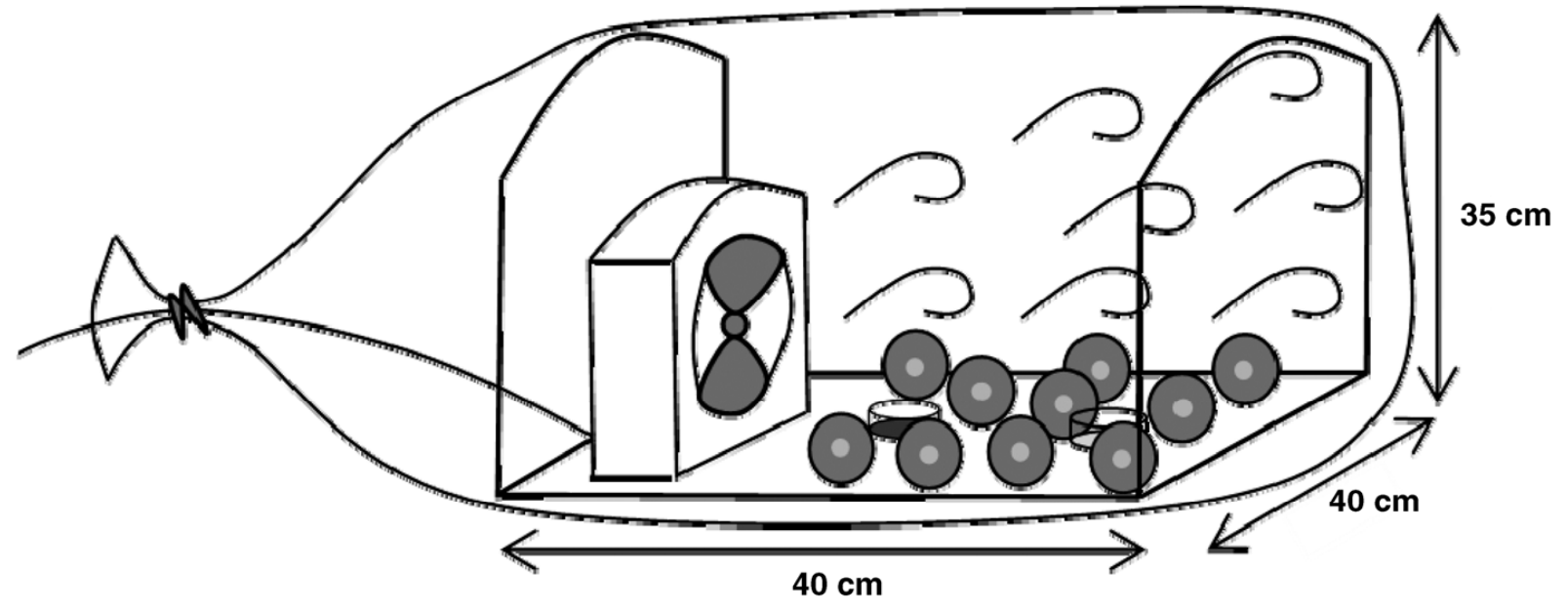

Penicillium culture $(65 \mathrm{~mm})$

Clean MEA plate

Fruit with $5 \mathrm{~mm}$ wounds

\section{Fan}

Fig. 1. Experimental setup for air inoculation of fruit in a mini-air chamber. 
spp. isolates and control (group c). It was again noted that $P$. crustosum and $P$. expansum were able to cause tissue-response lesions on Nules Clementine. Lesions caused by pear isolates were $2.1 \pm 0.7 \mathrm{~mm}(10.5 \%)$ and $2.4 \pm 1.1 \mathrm{~mm}$ (10.5\%), respectively. Lesions caused by citrus isolates were $4.6 \pm 1.7 \mathrm{~mm}(41.2 \%)$ and $2.1 \pm 0.7 \mathrm{~mm}(11.1 \%)$, respectively. The mean of control wounds had already been subtracted from the lesion diameters.

Comparison of inoculation methods. Results from the independent experiments were pooled $(P=0.6)$. The mean lesion sizes produced by the inoculation methods differed significantly $(P<$ 0.0001). The plug method received a separate t-grouping from the conidial suspension and air inoculation methods. However, the mean of lesions caused by $P$. digitatum from inoculation via conidial suspension was significantly different from inoculation via air but not plugs. Decay lesions caused by $P$. expansum and $P$. crustosum were on Eureka seeded when inoculation took place via the plug method (Figs. 2 and 3). Lesions caused by P. expansum and $P$. crustosum were characterized by rapid initial growth rates but rates decreased as decay progressed. The other methods were only able to facilitate tissue-response lesions from $P$. expansum and $P$. crustosum inoculations (Fig. 3). Inoculation via air (cultured plate as source of inoculum) delivered one lesion caused by $P$. crustosum $(1.1 \mathrm{~mm})$ and six lesions caused by $P$. expansum $(3.9 \pm 5.4 \mathrm{~mm})$. Inoculation via conidial suspensions delivered a single lesion caused by $P$. expansum $(2.4 \mathrm{~mm})$. The means of control wounds were already subtracted. Some lesions were small but the inoculated species were able to sporulate (Fig. 3).

Inoculations via conidial suspensions were found to be convenient and less time consuming but results produced varied more compared with plug inoculation. Inoculation via plugs delivered the highest incidence $(P$. digitatum and $P$. italicum $=100.0 \%, P$. crustosum $=90.0 \%$, and $P$. expansum $=94.9 \%$ ), measurements from replicates deviated the least, symptom expression was rapid and well-defined, lesions caused by $P$. italicum were significantly larger, and it was the only method facilitating $P$. crustosum and $P$. expansum to produce prominent decay lesions on citrus (Figs. 2 and 3). Inoculation via air was the most sensitive to contamination and least convenient compared with the other methods. In addition, inoculation via air also revealed biased results when directly using cultures as a source of inoculum (can also be expected form inocu- lation via plugs). Disease incidence of $P$. digitatum was low $(20.0 \%)$ when inoculated via air and using plates as source of inoculum. Upon completing air inoculations using an infected lemon covered with conidia ( 7 days ambient incubation) as source of inoculum, a $P$. digitatum disease incidence of $82.5 \%$ was achieved. This incidence was comparable with that achieved with inoculations via conidial suspension $(84.2 \%)$ and the mean of the lesion size was still similar to that obtained from inoculation via air using a $P$. digitatum culture plate as source of inoculum $(76.4 \pm 19.9$ versus $79.2 \pm 18.9 \mathrm{~mm}$ ).

Penicillium decay of lemon fruit under cold storage conditions. Lesion development was highly significantly influenced by temperature. The individual experiments for the cold-storage trial did not differ significantly on the 7-day incubation period $(P=$ $0.5)$. However, contamination emerged within the third week of the first experiment (predominantly only fruit stored at cold conditions). Only results from the second experiment will be discussed. $P$. digitatum and $P$. italicum were able to cause lesions under coldstorage conditions $\left(5.0 \pm 0.7^{\circ} \mathrm{C}\right.$ and $\left.86.4 \pm 4.5 \% \mathrm{RH}\right)$. The lesions were $43.8 \pm 5.6$ and $19.9 \pm 6.9 \mathrm{~mm}$, respectively, after 26 days of cold storage and $96.2 \pm 16.3$ and $33.9 \pm 11.0 \mathrm{~mm}$, respectively, after 7 days of ambient storage (mean of control wounds already subtracted). No lesions were observed from P. crustosuminoculated lemon fruit stored at any condition. A single tissueresponse lesion caused by $P$. expansum developed under ambient conditions after 7 days of incubation $(l d=6.9 \mathrm{~mm})$.

Lesion growth rates were calculated from the first day of lesion development to the last day of measurement. Growth rates at ambient and cold conditions for $P$. digitatum were 13.8 and $3.4 \mathrm{~mm} /$ day, respectively, and, for $P$. italicum, were 4.8 and $1.4 \mathrm{~mm} /$ day, respectively. The growth rate of lesions caused by $P$. digitatum and $P$. italicum was correspondingly reduced by 75.5 and $70.7 \%$, respectively, due to the cold conditions. The largest lesions at cold storage ( $43.8 \pm 15.6$ and $19.9 \pm 6.9 \mathrm{~mm}$, respectively) were delayed by 21 and 20 days, respectively. The first signs of lesion development under cold conditions from $P$. digitatum-inoculated fruit was observed on day 13 to 14 , whereas P. italicum started to cause lesions a day earlier. Alternatively, $P$. digitatum was the first to cause lesions under ambient conditions (day 1 to 2 ) and P. italicum only a day thereafter. The earliest mycelia and conidia were observed on

Table 2. Pathogenicity and disease intensity of Penicillium spp. on citrus

\begin{tabular}{|c|c|c|c|c|}
\hline Cultivar, species & Lesions $(\mathrm{mm})^{\mathrm{x}}$ & Incidence $(\%)^{\mathrm{y}}$ & Significant lesions $\mathbf{s}^{\mathbf{x}, \mathbf{y}}$ & Disease intensity $(\%)^{\mathrm{z}}$ \\
\hline \multicolumn{5}{|l|}{ Nules Clementine } \\
\hline Penicillium digitatum & $84.2 \pm 21.8 \mathrm{a}$ & 100.0 & $84.2 \pm 21.8$ & 91.3 \\
\hline P. italicum & $34.3 \pm 16.4 b$ & 80.0 & $35.9 \pm 15.5$ & 31.1 \\
\hline P. crustosum & $2.6 \pm 2.8 \mathrm{c}$ & 5.6 & 9.1 & 0.6 \\
\hline P. expansum & $1.0 \pm 2.0 \mathrm{c}$ & 5.0 & 10.7 & 0.6 \\
\hline Control & $0.1 \pm 0.2 \mathrm{c}$ & - & - & - \\
\hline \multicolumn{5}{|l|}{ New Hall Navel } \\
\hline P. digitatum & $81.0 \pm 26.8 \mathrm{a}$ & 95.0 & $84.9 \pm 21.2$ & 78.2 \\
\hline P. italicum & $33.1 \pm 7.8 \mathrm{~b}$ & 79.0 & $33.1 \pm 7.8$ & 25.4 \\
\hline P. crustosum & $1.1 \pm 2.6 \mathrm{c}$ & 0 & - & - \\
\hline P. expansum & $0.9 \pm 1.8 \mathrm{c}$ & 0 & - & - \\
\hline Control & $0.1 \pm 0.2 \mathrm{c}$ & - & - & - \\
\hline \multicolumn{5}{|l|}{ Star Ruby } \\
\hline P. digitatum & $119.8 \pm 20.7 \mathrm{a}$ & 90.0 & $119.8 \pm 20.7$ & 81.3 \\
\hline P. italicum & $32.1 \pm 9.6 \mathrm{~b}$ & 85.0 & $32.1 \pm 9.6$ & 20.6 \\
\hline P. crustosum & $2.4 \pm 2.5 \mathrm{c}$ & 16.7 & $7.9 \pm 0.5$ & 1.0 \\
\hline P. expansum & $1.4 \pm 1.5 \mathrm{c}$ & 5.0 & 9.3 & 0.4 \\
\hline Control & $0.1 \pm 0.2 \mathrm{c}$ & - & - & - \\
\hline \multicolumn{5}{|l|}{ Eureka seeded } \\
\hline$P$. digitatum & $84.4 \pm 10.9 \mathrm{a}$ & 90.0 & $84.4 \pm 10.9$ & 84.9 \\
\hline P. italicum & $46.4 \pm 6.9 b$ & 85.0 & $46.4 \pm 6.9$ & 44.0 \\
\hline P. crustosum & $0.4 \pm 1.1 \mathrm{c}$ & 0 & - & - \\
\hline P. expansum & $0.1 \pm 0.1 \mathrm{c}$ & 0 & - & - \\
\hline Control & $0.1 \pm 0.1 \mathrm{c}$ & - & - & - \\
\hline
\end{tabular}

\footnotetext{
${ }^{\mathrm{x}}$ Mean of the control diameters was subtracted from the mean of the measured diameters. Means followed by \pm standard deviation.

${ }^{y}$ Only measurements significantly larger than the mean of the control diameter were included in calculating figures in this column.

${ }^{\mathrm{z}}$ Disease intensity $=\left[(d \times F) / T_{\mathrm{n}} \times D\right] \times 100 . D$ values: Nules Clementine $=92.25 \mathrm{~mm}$, New Hall Navel $=103.13 \mathrm{~mm}$, Star Ruby $=132.51 \mathrm{~mm}$, and Eureka seeded $=89.55 \mathrm{~mm}$. Figures from incidence and significant lesions columns were used in the equation.
} 
day 21 to 22 under cold-storage conditions versus day 4 to 5 under ambient conditions for both $P$. digitatum and $P$. italicum.

Penicillium spp. aggressiveness on citrus. Results from independent experiments were not significantly different and thus pooled $(P<0.0001)$. The interaction effect of the Penicillium spp. on the different cultivars showed significant difference $(P<$ $0.0001)$. Large lesions were caused by $P$. digitatum and $P$. italicum over the whole citrus range evaluated (Fig. 4). However, lesion sizes produced did vary significantly. In general, the lesions caused by $P$. digitatum decreased in size: lemon $>$ mandarin $>$ sweet orange $>$ grapefruit. Aggressiveness of $P$. digitatum varied more over mandarin cultivars (each cultivar grouped in separate t-groupings) and less over sweet orange cultivars (some Navel and Valencia did not differ significantly). The aggressiveness of $P$. italicum was more consistent over the citrus range than that of $P$. digitatum. The means of almost all lesions caused by $P$. italicum were grouped together or in related t-grouping, except for those on Owari Satsuma and Cambria Navel. The largest mean of lesion diameters caused by $P$. digitatum and $P$. italicum was on Eureka seeded and Owari Satsuma, respectively.

The means of lesions caused by $P$. crustosum and $P$. expansum were not significantly different compared with the control on the fifth day of incubation (Fig. 4). However, some lesions caused by $P$. crustosum and P. expansum (decay and tissue-response lesions) were significantly different on the seventh day of incubation. Most lesions were small and developed at low incidences (Table 3).

Penicillium symptom expression on citrus cultivars. Additional symptom characteristics caused by the Penicillium spp. were distinguished on the citrus range evaluated. Lesions caused by $P$. digitatum and $P$. italicum radiated with a watery soaked appearance as infections progressed. Infected rind (pericarp) tissue lost its smoothness and became more susceptible to mechanical damage. $P$. italicum exhibited darker infected tissue on mandarin cultivars and Eureka seeded than on sweet orange cultivars and Star Ruby (darkening more localized around the inoculation sites; Fig. 5). White mycelial growth later started to radiate from the infected tissue, followed by sporulation (dark-olive-green conidia for $P$. digitatum and blue conidia for $P$. italicum).

Decay lesions caused by $P$. crustosum and $P$. expansum on Eureka seeded (plug inoculated) and some mandarin cultivars (conidial suspension inoculated) were sunken in appearance and infected (affected) rind tissue was harder, drier, and browner than healthy tissue. This only diverged with Nules Clementine and Owari Satsuma, in which cases the lesions were softer than mentioned before. Lesions of P. crustosum were, in general, lighter colored (Fig. 3) but not consistently over the tested citrus range (Fig. 5). The browning of lesions varied, depending on numerous factors (Penicillium spp., cultivar host and presumably environmental factors). Tissue-response lesions (typical HR reactions) were characterized as small, hardened, dark, and sunken symptoms. Some HR reactions became relatively large compared with typical symptoms characteristics expected from such reactions (Table 3; Fig. 3).

Mycelial growth and sporulation of $P$. crustosum and $P$. expansum from decay lesions were occasionally restricted to close proximity of the inoculated sites. Both species were also able to sporulate at some tissue-response lesions, despite the small size of the lesions (Figs. 3 and 5). This caused difficulties when trying to characterize such lesions, especially when they were also significantly larger than controls. In the case of inoculation via plugs, sporulation of $P$. crustosum from decay lesions were limited to close proximity of the inoculated site when observing the lesions from the fruit surface (on the exocarp). However, underneath the exocarp, sporulation took place within the spongy mesocarp and endocarp of infected pericarp tissue. Even fruit pulp or juice vesicles were affected (browning). Sporulation underneath the exocarp of infected $P$. expansum lemon fruit was not observed, although the infected rind tissue was drier and harder than $P$. crustosum-infected tissue (Fig. 3). P. crustosum and $P$. expansum produced mycelia and sporulated from some lesions on Nules Clementine (P. crustosum mycelial growth on day three and sporulation on day five; $P$. expansum mycelial growth on day five and sporulation day seven), and Owari Satsuma (P. crustosum mycelial growth and sporulation

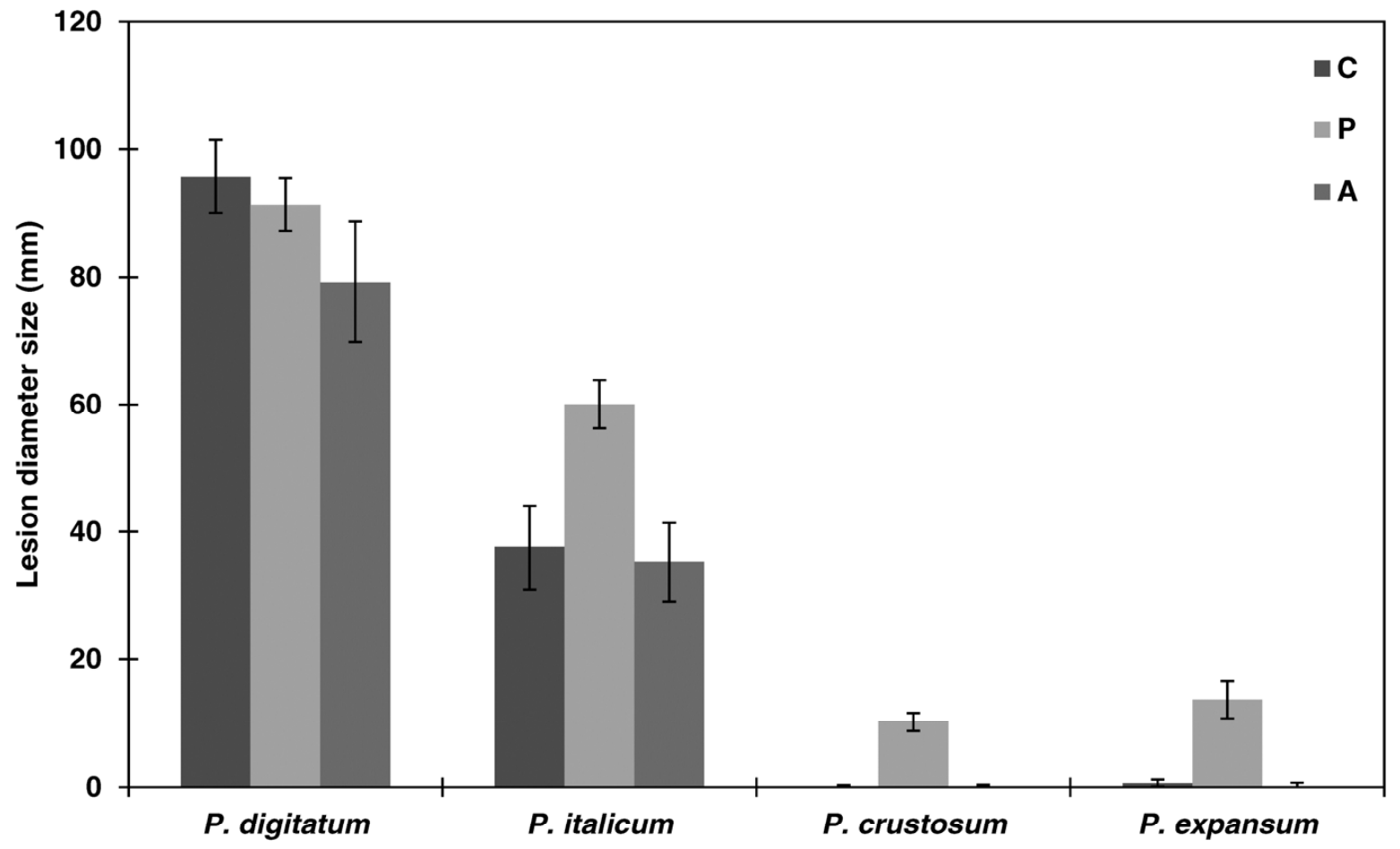

Penicillium spp.

Fig. 2. Seven-day lesion diameters of Penicillium spp. inoculated into Eureka seeded using three different inoculation methods $\left(21.9 \pm 0.4^{\circ} \mathrm{C}\right.$ and $43.6 \pm 4.6 \%$ relative humidity): $\mathrm{C}=$ inoculation via conidial suspensions (conidial suspension inoculation moving average), $\mathrm{P}=$ inoculation via plugs (plug inoculation moving average), and $\mathrm{A}=$ inoculation via air (air inoculation moving average). Bars illustrate standard deviation. 


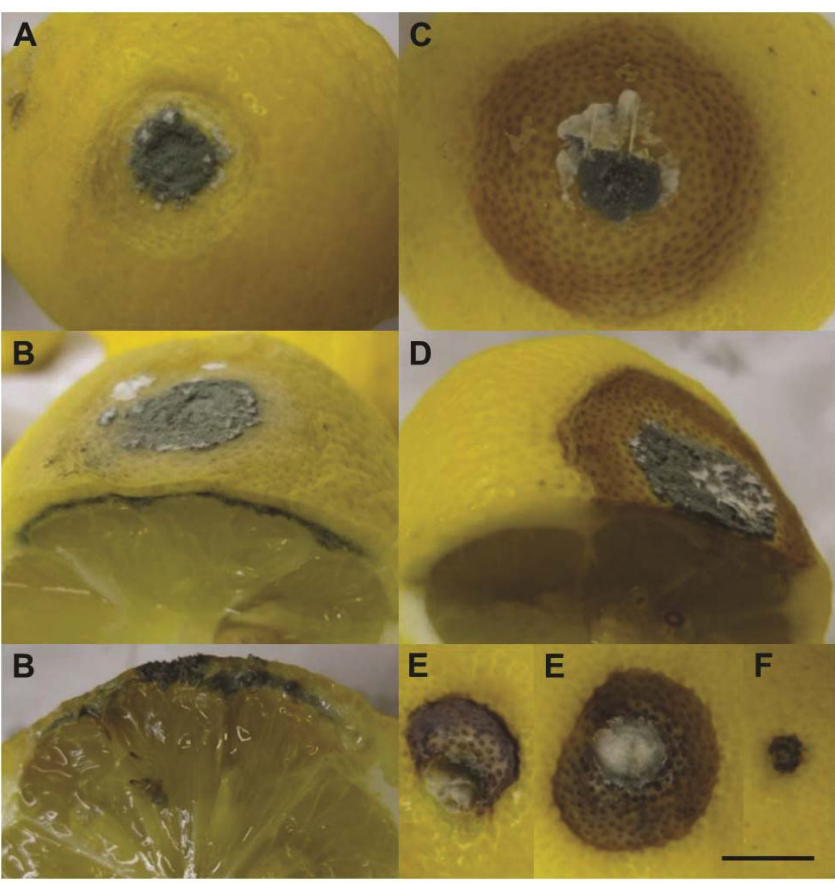

Fig. 3. Lesions and infection reactions caused by Penicillium crustosum and $P$. expansum on Eureka seeded inoculated via three different methods: $\mathbf{A}$ and $\mathbf{B}, 7$ thday $P$. crustosum lesions via plug method; $\mathbf{C}$ and $\mathbf{D}, 7$ th-day $P$. expansum lesions via plug method; $E$, 14th day $P$. expansum lesions via air method; and $F$, 14th day $P$. expansum lesions via suspension method. $B a r=10 \mathrm{~mm}$. on day four to five; $P$. expansum mycelial growth and sporulation on day six to seven). Only $P$. crustosum produced mycelia and conidia on Nova (day two to three) and Delta Valencia (day six to seven). Conidia produced by $P$. crustosum were pale turquoise number 4 and conidia from $P$. expansum were light blue number 4 with a tint grayer in color (Figs. 3 and 5).

The first signs of $P$. digitatum and $P$. italicum mycelial growth and sporulation were detected on day four to five on nearly all cultivars inoculated. It was only detected earlier on Nules Clementine (day three). The color or shade of conidia produced from the lesions was not continuously uniform over the citrus range. The shade of green of the $P$. digitatum conidia varied. Cambria Navel, Midknight Valencia, Delta Valencia, and Star Ruby displayed conidia of dark olive-green number 4. New Hall Navel, Palmer Navel, and Nova resulted in the production of dark olive-green conidia. Conidia produced by $P$. digitatum from lesions on Owari Satsuma and Eureka seeded were dark olive-green with a tint of more gray and, finally, conidia from lesions on Nules Clementine were dark sea-green number 4 in color. $P$. italicum conidial color was more consistent, ranging from light blue number 3 to light blue number 4 , depending on the amount of conidia grouped (Figs. 3 and 5).

Isolate identity confirmation. Reisolated Penicillium spp. from the infected sites were confirmed as the previously inoculated species by PCR-RFLP and sequencing. The $\beta$-tubulin gene sequences were submitted in GenBank (Table 4).

\section{Discussion}

$P$. crustosum and $P$. expansum were pathogenic on some cultivars with varying incidence and aggressiveness. Both species

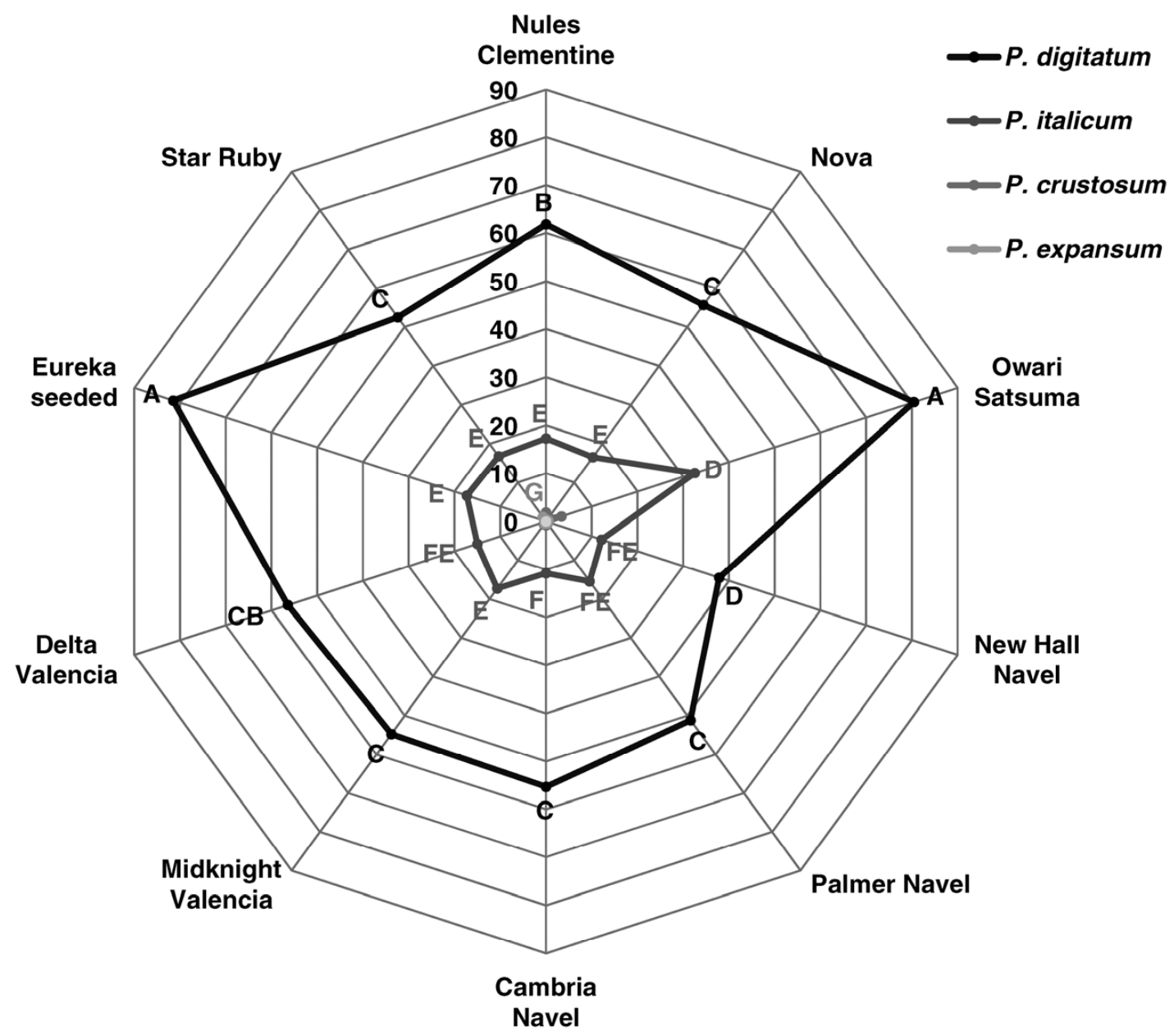

Fig. 4. Lesion and infection reaction diameters $(\mathrm{mm})$ caused by Penicillium spp. infecting citrus cultivars (incubated under ambient condition for 5 days). Letters that are not the same are significantly different. 
caused decay lesions on lemon fruit and mandarin fruit but only tissue response lesions on most of the remaining cultivars evaluated. Conidia were produced on decay and some of the tissueresponse lesions (Figs. 3 and 5), allowing P. crustosum and P. expansum to complete their life cycles in these cases. Additionally, the small lesions will affect marketability of fruit. These species are well-known deciduous fruit pathogens (16) but very few reports have described them as citrus pathogens and none has described the species as pathogens on a broad citrus host range or provided complete symptom descriptions $(2,10,17,34)$. This also links citrus with the potential harmful toxins associated with the species: citrinin (nephrotoxin), communesin B (cytotoxic), patulin (multiple range), penitrem $\mathrm{A}$ (neurotoxin), roquefortine $\mathrm{C}$ (neurotoxin), terrestric acid (cardiotoxin), and others $(8,9,21)$.

Garcha and Singh (10) conducted cross-inoculation studies, revealing complete $P$. crustosum decay of mandarin fruit within 8 days. This was the first report demonstrating that $P$. crustosum is pathogenic on citrus. They confirmed Koch's postulates and identified $P$. crustosum via morphological techniques used at that time. Symptoms described were similar to those reported in this study (shallow or sunken lesions, mycelial growth as clumps, and the bluish-green color of conidia). The fermented odor noted by Garcha and Singh (10) was not observed in this study and the symptom illustrations could not be compared due to the vagueness of their images. A more recent study concerning P. crustosum on citrus reported a mean lesion diameter of $17.3 \pm 5.2 \mathrm{~mm}$ at $36.0 \%$ incidence (3). Their study focused mainly on biocontrol screening and, therefore, did not elaborate on the lesions produced by $P$. crustosum.

Our findings confirmed $P$. crustosum as pathogenic on mandarin fruit (10) but only tissue-response lesions were noted on Valencia orange fruit (3). Lesions were also smaller and disease incidence lower. Differences with Garcha and Singh (10) on mandarin fruit can be ascribed to different incubation conditions (higher $\mathrm{RH}$ of $90 \%$, temperature unmentioned), inoculum concentration (not specified), inoculation method (cotton pad wetted with conidial suspension and placed in a pinpricked fruit for $48 \mathrm{~h}$ ), fruit age (market fruit), and cultivar. Differences noted by Arrebolla et al. (3) on Valencia are likewise credited to different incubation conditions (higher temperature of $25^{\circ} \mathrm{C}$, humidity unspecified), inoculum concentration (higher inoculum concentration of $3 \times 10^{6}$ conidia/ml), inoculation method (dipping of wounded fruit in conidial suspension for $3 \mathrm{~min}$ ), and incubation period (12 days). Vilanova et al. (34) have shown that conidial concentrations can affect lesion growth rate, initial lesion development, and disease incidence. Disease incidence can be influenced by inoculum concentration to such an extent that certain pathogens can be overlooked if inoculum concentrations are too low. Our study is the first to report P. crustosum as pathogenic on Nules Clementine,

Table 3. Citrus-Penicillium spp. disease interactions with incomplete incidence $(<100 \%)$ after 7 days of incubation

\begin{tabular}{lcc}
\hline Species, cultivar & $\begin{array}{c}\text { Mean of significant } \\
\text { lesions }^{\mathbf{z}}\end{array}$ & $\begin{array}{c}\text { Incidence } \\
(\boldsymbol{\%})\end{array}$ \\
\hline Penicillium crustosum & $9.1 \pm 2.9$ & \\
Nules Clementine & $11.9 \pm 1.7$ & 29.7 \\
Nova & $11.0 \pm 2.9$ & 10.8 \\
Owari Satsuma & $6.0 \pm 0.7$ & 48.3 \\
Delta Valencia & 7.1 & 8.8 \\
Cambria Navel & $7.9 \pm 0.5$ & 2.6 \\
Star Ruby & & 8.1 \\
P. expansum & $13.3 \pm 1.1$ & 6.1 \\
Nules Clementine & $9.0 \pm 1.7$ & 19.4 \\
Owari Satsuma & $7.7 \pm 0.4$ & 7.1 \\
Midknight Valencia & 14.8 & 2.6 \\
Delta Valencia &
\end{tabular}

${ }^{\mathrm{z}}$ Only measurements significantly larger than the mean of the control diameter were included in calculating these figures. Mean of the control diameters were subtracted from the mean of the measured diameters. Means followed by \pm standard deviation.
Nova, Owari Satsuma, and Eureka seeded. It is also the first report of tissue-response lesions caused by $P$. crustosum on Delta Valencia, Cambria Navel, and Star Ruby. Symptoms on the mandarin cultivars and Eureka seeded were well-documented.

Macarisin et al. (17) inoculated lemon, grapefruit, and orange fruit (cultivars not mentioned) with $20 \mu \mathrm{l}$ of $P$. digitatum and $P$. expansum conidial suspensions $\left(10^{5}\right.$ conidia/ml $)$. Fruit were incubated at $20^{\circ} \mathrm{C}$ in darkness in covered plastic trays with moistened filter paper. They found that $P$. expansum was able to germinate and temporarily grow in the peel wounds of the three inoculated citrus groups. Growth progressed until the plant defense-related oxidative burst was triggered, leading to HR, averting infection or invasion (17). They additionally reported that $P$. digitatum is able

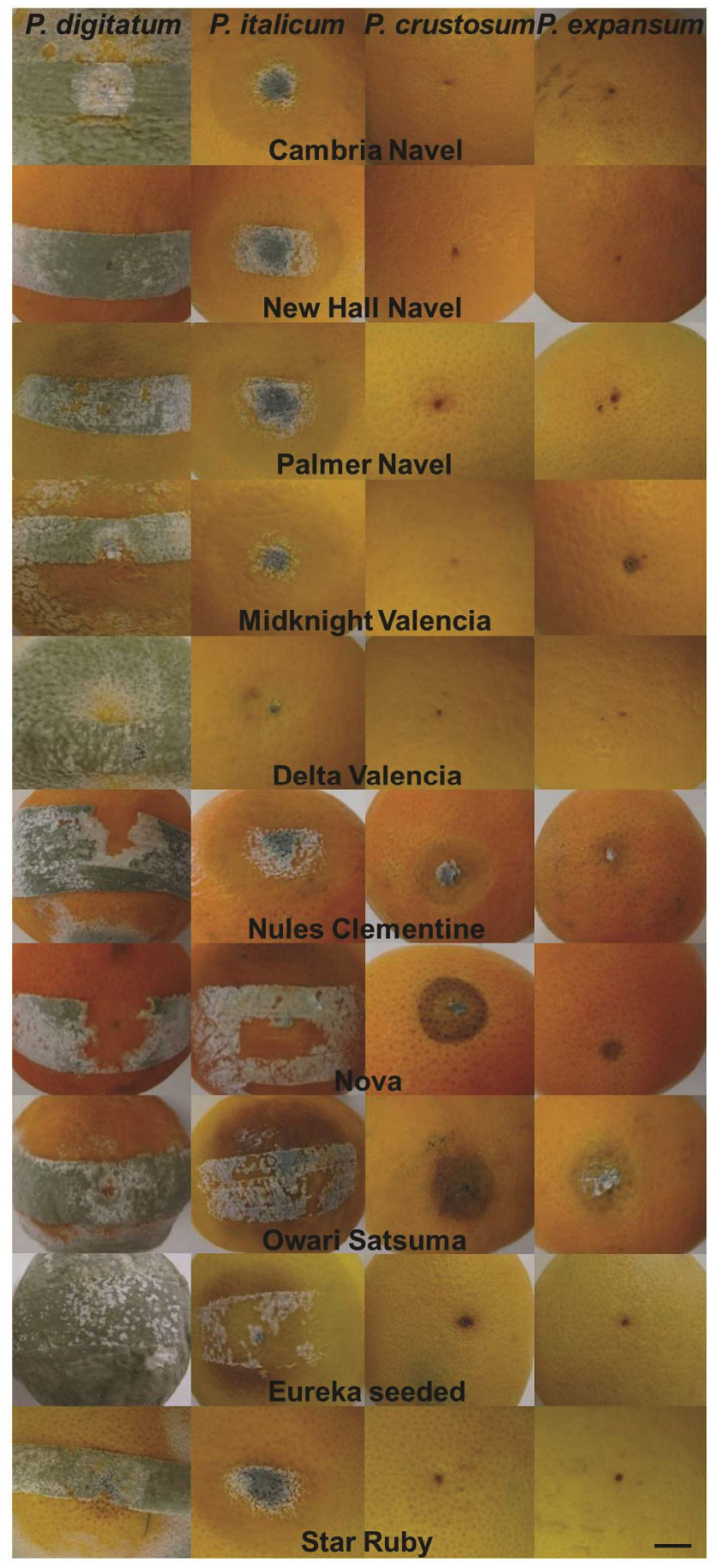

Fig. 5. Symptom expression of Penicillium spp. (columns from left to right: Penicillium digitatum, $P$. italicum, $P$. crustosum, and $P$. expansum) on citrus after 7 days of incubation at ambient temperatures. $B a r=10 \mathrm{~mm}$. 
to infect citrus because the species is able to suppress the host's defense-related oxidative burst. Based on this, Macarisin et al. (17) treated fruit with citric, ascorbic, and oxalic acids and enzyme catalase (suggestive $\mathrm{H}_{2} \mathrm{O}_{2}$ production suppressors) before inoculation with $P$. expansum. This resulted in lesions caused by $P$. expansum on all three citrus groups. They were the first to report on the pathogenic potential of $P$. expansum on citrus, although lesions produced by the nonhost pathogen were artificially stimulated. No symptom descriptions were provided for the lesions.

Vilanova et al. (34) were able to observe well-defined lesions caused by $P$. expansum on citrus (Valencia and Navelina orange) without employing chemical pretreatments such as those used by Macarisin et al. (17). Rot caused by $P$. expansum was only observed after inoculating fruit with high conidial concentrations (no lesions at $10^{5}$ or $10^{4}$ conidia $\left./ \mathrm{ml}\right)$. Lesion diameters were $3 \mathrm{~mm}\left(10^{6}\right.$ conidia/ml $)$ and $8 \mathrm{~mm}\left(10^{7}\right.$ conidia/ml $)$ after 17 days of incubation $\left(20^{\circ} \mathrm{C}, 85 \% \mathrm{RH}\right)$. Much larger lesions were recorded on Navelina: up to $35 \mathrm{~mm}$ in diameter within 11 days. The more suitable RH for decay could have contributed to advanced lesion size $(2,27)$ and symptom development (mycelial growth and sporulation). Vilanova et al. (34) additionally reported orange-red-colored reactions around inoculation sites on the flavedo when $P$. expansum was unable to infect. The albedo tissue underneath the inoculated sites was dead. The reactions were concentration dependent and prominent when inoculating fruit with high inoculum concentrations $\left(10^{7}\right.$ conidia/ml). No reactions were observed from fruit inoculated with $10^{4}$ conidia/ml. This was the first report where $P$. expansum caused decay of orange fruit under specific conditions.

We observed lesions on Valencia but not on Navel orange fruit after inoculation with $P$. expansum (34). The lower inoculum concentrations used in our study may have been too low to facilitate infections. Our study is the first to report $P$. expansum as pathogenic on lemon fruit (Eureka seeded) when inoculated via plugs (high concentration) and mandarin fruit (Nules Clementine and Owari Satsuma) when inoculated via conidial suspensions (low concentration). It is also the first report of tissue-response lesions caused by $P$. expansum recorded on Midknight Valencia and Delta Valencia after direct inoculation with a low inoculum concentration $(6.4$ $\times 10^{4}$ conidia $/ \mathrm{ml}$ ). Symptoms on Eureka seeded and mandarin fruit in our study presented similarity to symptoms illustrated by Vilanova et al. (34) on Valencia and Navelina orange fruit.

The well-known postharvest citrus pathogens $P$. digitatum and $P$. italicum (12) were confirmed as being the most aggressive Penicillium spp. on all the citrus cultivars in terms of decay and incidence. Lesions caused by $P$. digitatum decreased in size from mandarin fruit and Eureka seeded to sweet orange and Star Ruby, confirming citrus susceptibility increasing from grapefruit to orange, lemon, and mandarin fruit for most diseases (6). This was not observed with $P$. italicum, which expressed itself as a general pathogen over the entire citrus range evaluated in this study.

Aggressiveness of $P$. digitatum varied more over mandarin than over sweet orange cultivars. This could be due to a larger variance found among the mandarin cultivars tested. The mandarin cultivars differed on species level (according to Tanaka classification;

Table 4. Accession numbers (GenBank) of $\beta$-tubulin gene sequences

\begin{tabular}{llc}
\hline Isolate number & \multicolumn{1}{c}{ Species identity } & Accession number \\
\hline C57 & Penicillium digitatum & KF952540 \\
C55 & P. digitatum & KF952539 \\
C51 & P. digitatum & KF952538 \\
C45 & P. italicum & KF952537 \\
C41 & P. italicum & KF952536 \\
C37 & P. italicum & KF952535 \\
C29 & P. crustosum & KF952534 \\
C27 & P. crustosum & KF952533 \\
C23 & P. crustosum & KF952532 \\
C15 & P. expansum & KF952531 \\
C11 & P. expansum & KF952530 \\
C6 & P. expansum & KF952529 \\
\hline
\end{tabular}

28,29), whereas sweet orange cultivars all belonged to the same Citrus sp. Owari Satsuma was expected to be the most susceptible mandarin cultivar (industry observation) but, in our study, the fruit were stored for a prolonged period, which could have contributed to the larger lesions observed. Larger lesions caused by $P$. digitatum and P. italicum were expected on Navel than on Valencia orange fruit (industry observation) but this was not observed. The smallest lesions were observed on New Hall Navel and the largest on Delta Valencia in this study. This can be based on differing incubation environments but susceptibility alone depends on multiple factors. Susceptibility varies among cultivars and can be influenced by scion wood or rootstocks, cultural practices, harvest season, water and nutrient status of tree, fruit maturity, and the postharvest environment (6).

Symptoms recorded in this study added descriptions to rather undescribed $P$. crustosum and $P$. expansum symptoms $(10,34)$. Symptoms of $P$. digitatum and $P$. italicum are well known $(7,25)$ but not necessarily over a range of citrus cultivars. Little $P$. italicum symptom variance was observed over the citrus ranges: conidial color was relatively constant but lesion darkness and time required for initial mycelial growth and sporulation differed to some level. Regarding $P$. digitatum, lesion darkness was more constant over the citrus range but this was not the case with conidial color.

Different isolates (citrus- and pear-chain isolates) were not significantly different when lesion sizes were compared, indicating that disease severity was not significantly affected by the type of isolate. However, decay problems should be connected to the most likely origin of the inoculum sources (i.e., confinements with high inoculum loads) so that problem areas can be highlighted in the supply chain. Introduction of $P$. crustosum and $P$. expansum conidia from pome fruit into an environment where citrus fruit are also handled or repacked can result in cross-contamination and crossinfection of citrus fruit, especially when inoculum levels are high (34). The probability of cross-infection of $P$. digitatum from citrus to pome fruit has also been projected by Louw and Korsten (16). This aspect is important because different fruit types are often handled in the same environment during periods of overlapping seasons and toward the end of the chain (i.e., citrus and pome fruit).

Results from inoculation via plugs were the most consistent and symptoms were well defined. However, the inoculation method represents the most unlikely pathway of natural infection in orchards or within the handling and packing environments. The welldescribed spread of infections among fruit that contact each other is one of the few natural scenarios illustrating potential infection via this method (13). The unique results produced with the plug method can be attributed to the direct inoculation of concentrated inoculum in an open wound (34). The role that inoculum load plays in incompatible host-pathogen interactions (nonhost pathogens) has been highlighted on citrus (34) and apple (32,33).

The inoculation of fruit via aerially dispersed conidia is the most likely pathway for natural Penicillium infections. This method still requires further modifications before it can be regarded as a suitable postharvest inoculation method for inoculating fruit or vegetables with pathogens capable of air dissemination. The method was found to be the least convenient and most sensitive to crosscontamination and the inoculum source in this study. The culturing of Penicillium spp. on artificial media can result in advanced growth or sporulation for certain species (i.e., $P$. expansum and $P$. crustosum grew faster and sporulated more abundantly than $P$. digitatum on MEA within the same incubation period and conditions). Sensitivity of the method to contamination was overcome by inoculating fruit in the inoculation chamber set up inside a biosafety cabinet $(14,23)$. An easier-to-sterilize inoculation chamber and making use of infected fruit as source of inoculum are proposed improvements. Fruit as source of inoculum serve as a more natural infection source and pathway, and remove the biased interaction found with culturing. Alternative to infected fruit as a source of inoculum, dry conidial masses (conidia per gram) can be used to standardize inoculum loads disseminated in chambers. Specifying 
atmospheric inoculum loads (conidia per cubic centimeter) required for each species to cause decay will prove beneficial for industry.

Despite the advantages associated with inoculation via plugs or air, inoculation via conidial suspensions was still regarded as the most suitable inoculation method for citrus at the time. Improvement for inoculation via air may lead to the method being preferred for Penicillium inoculation studies.

Environmental conditions affected $P$. digitatum and $P$. italicum aggressiveness and symptom expression. $P$. digitatum caused the largest lesions, and $P$. expansum and $P$. crustosum caused no lesions under the cold conditions. Vilanova et al. (34) reported large lesions caused by $P$. expansum on orange fruit (Valencia $l d= \pm 45$ $\mathrm{mm}$ and Navelina $l d=70$ to $100 \mathrm{~mm}$ ) after 75 days of incubation under cold-storage conditions $\left(4^{\circ} \mathrm{C}\right)$. This was only achieved when fruit were inoculated with concentrations of $10^{6}$ and $10^{7}$ conidia $/ \mathrm{ml}$ and not $10^{4}$ and $10^{5}$ conidia/ml. Our results support the finding that low inoculum concentrations were unable to cause lesions under cold storage on citrus (34). However, the lower concentrations (6.3 $\times 10^{4}$ conidia $/ \mathrm{ml}$ ) were able to cause lesions on apple fruit under cold storage (16), demonstrating that $P$. expansum and $P$. crustosum are opportunistic pathogens of citrus.

This study has highlighted the importance of controlling inoculum levels within the fruit chain. Understandably, control practices have been established to control or attempt to control a broad postharvest Penicillium pathogen range but industry has not seriously considered the formerly nonhost pathogen, P. crustosum and $P$. expansum, a concern on citrus. The disregard or lack of attention to prevent or lower the increase of inoculum levels of nonhost pathogens in the fruit chain may pose a risk to industry. Thus, the handling and storage of different fruit species within the same environment (31) may lead to increased inoculum levels of these pathogens, thereby increasing inoculum pressure and contributing to decay (34). Future studies should investigate the link among market-end losses, the casual agents involved, and inoculum levels and sources.

Conclusion. All tested species ( $P$. digitatum, $P$. italicum, $P$. crustosum, and $P$. expansum) were pathogenic on citrus, although pathogenicity and aggression varied over the citrus range. $P$. digitatum and $P$. italicum were pathogenic over the entire citrus range, exhibiting high levels of aggression. Specific conditions and cultivars were required for $P$. crustosum and $P$. expansum to express decay, high aggression, and proper symptom development. The species were regarded as opportunistic pathogens on citrus, dependent on the inoculum levels, inoculation pathway, host susceptibility, and environmental conditions (cold-chain management). This is the first report demonstrating decay lesions caused by $P$. crustosum on Nules Clementine, Nova, Owari Satsuma, and Eureka seeded, and tissue-response lesions on Delta Valencia, Cambria Navel, and Star Ruby. It is also the first report of decay lesions caused by $P$. expansum on Nules Clementine, Owari Satsuma, and Eureka seeded, and tissue-response lesions on Midknight Valencia and Delta Valencia. Additionally, varying aggression of $P$. digitatum and $P$. italicum over a broad citrus host range and further symptom descriptions on citrus-Penicillium infections have been reported in this study. Varying aggression over the citrus range displayed Citrus spp. and cultivar-related Penicillium decay concerns in the fruit industry, thus highlighting areas requiring additional attention. Future studies should focus on market-end fruit susceptibility and inoculum loads further down the fruit chain.

\section{Acknowledgments}

The work is based on the research supported, in part, by a number of grants from the National Research Foundation of South Africa UID: 78566 (NRF RISP grant for the ABI3500) and student support. The grant holders acknowledge that opinions, findings, and conclusions or recommendations expressed in any publication generated by the NRF-supported research are those of the authors and that the NRF accepts no liability whatsoever in this regard. We thank R. Jacobs (Syngenta, Midrand, South Africa) for input with initial experimental design, method guidance, and general support; I. Scholtz for providing the Penicillium isolates; T. T. Ghebremariam for statistical support; Z. Zulu for molecular sup- port; L. Louw and S. B. Coetzee for trial assistance; W. J. Janisiewicz (United States Department of Agriculture-Agricultural Research Service, Kearneysville, WV) for editorial input; and Unifrutti South Africa (Kirkwood, Eastern Cape) for access to their fruit and laboratories.

\section{Literature Cited}

1. Agrios, G. N. 2005. Plant Pathology, 5th ed. Elsevier Academic Press, San Diego, CA.

2. Amiri, A., and Bompeix, G. 2005. Diversity and population dynamics of Penicillium spp. on apples in pre- and postharvest environments: Consequences for decay development. Plant Pathol. 54:74-81.

3. Arrebolla, E., Sivakumar, D., and Korsten, L. 2010. Effect of volatile compounds produced by Bacillus strains on postharvest decay in citrus. Biol. Control 53:122-128.

4. Barkai-Golan, R. 2001. Chemical control. Pages 147-188 in: Postharvest Diseases of Fruits and Vegetables: Development and Control. R. BarkaiGolan, ed. Elsevier Science B. V., Amsterdam, The Netherlands.

5. CGA. 2012. Key industry statistics for citrus growers 2012. Citrus Growers' Association of Southern Africa. http://www.cga.co.za

6. Eckert, J. W., and Eaks, I. L. 1989. Postharvest disorders and diseases of citrus Fruits. Pages 179-260 in: The Citrus Industry, Vol. 5, Revised ed. W. Reuther, E. C. Calavan, and G. E. Carman, eds. University of California, Division of Agricultural and Natural Resources, Publ. 3326, Richmond.

7. Fawcett, H. S., and Klotz, L. J. 1948. Diseases and their control. Pages 495 596 in: The Citrus Industry, Vol. 2: The Production of the Crop. L. D. Batchelor and H. J. Webber, eds. University of California Press, Berkeley.

8. Frisvad, J. C., and Samson, R. A. 2004. Polyphasic taxonomy of Penicillium subgenus Penicillium A guide to identification of food and air-borne terverticillate Penicillia and their mycotoxins. Stud. Mycol. 49:1-174.

9. Frisvad, J. C., Smedsgaard J., Larsen, T. O., and Samson, R. A. 2004. Mycotoxins, drugs and other extrolites produced by species in Penicillium subgenus Penicillium. Stud. Mycol. 49:201-241.

10. Garcha, H. S., and Singh, V. 1976. Penicillium crustosum, a new pathogen of Citrus reticulata (mandarin) from India. Plant Dis. Rep. 60:252-254.

11. Glass, N. L., and Donaldson, G. C. 1995. Development of primer sets designed for use with the PCR to amplify conserved genes from filamentous Ascomycetes. Appl. Environ. Microbiol. 61:1323-1330.

12. Holmes, G. J., and Eckert, J. W. 1999. Sensitivity of Penicillium digitatum and $P$. italicum to postharvest citrus fungicides in California. Phytopathology 89:716-721.

13. Kader, A. A. 2002. Postharvest Technology of Horticultural Crops, $3^{\text {rd }}$ ed. University of California Agricultural and Nutritional Resources Publ. 3311, Richmond.

14. Lee, J. H., Hwang, G. B., Jung, J. H., Lee, D. H., and Lee, B. U. 2009. Generation characteristics of fungal spore and fragment bioaerosols by airflow control over fungal cultures. J. Aerosol Sci. 41:319-325.

15. Liu, Y., Heying, E., and Tanumihardjo, S. A. 2012. History, global distribution, and nutritional importance of citrus fruits. Comp. Rev. Food Sci. Food Saf. 11:530-545.

16. Louw, J. P., and Korsten, L. 2014. Pathogenic Penicillium spp. on apples and pears. Plant Dis:98: 590-598.

17. Macarisin, D., Cohen, L., Eick, A., Rafael, G., Belausov, E., Wisniewski, M., and Droby, S., 2007. Penicillium digitatum suppresses production of hydrogen peroxide in host tissue infection of citrus fruit. Phytopathology 97:1491-1500.

18. Marcet-Houben, M., Ballester, A., De la Fuente, B., Harries, E., Marcos, J. F., González-Candelas, L., and Gabaldón, T. 2012. Genome sequence of the necrotrophic fungus Penicillium digitatum, the main postharvest pathogen of citrus. BMC Genomics 13:646.

19. Nunes, C., Duarte, A., Manso, T., Weiland, C., García, J. M., Cayuela, J. A., Yousfi, K., Martínez, M. C., and Salazar, M. 2010. Relationship between postharvest diseases resistance and mineral composition of citrus fruit. ISHS Acta Hortic. 868:417-422.

20. Pariaud, B., Ravigné, V., Halkett, F., Goyeau, H., Carlier, J., and Lannou, C. 2009. Aggressiveness and its role in the adaptation of plant pathogens. Plant Pathol. 58:409-424.

21. Pitt, J. I., and Hocking, A. D. 2009. Fungi and Food Spoilage, 3rd ed. Springer Science+Business Media, New York.

22. Plaza, P., Usall, J., Teixidó, N., and Viñas, I. 2003. Effect of water activity and temperature on germination and growth of Penicillium digitatum, $P$. italicum and Geotrichum candidum. J. Appl. Microbiol. 94:549-554.

23. Reponen, T., Willeke, K., Ulevicus, V., Grinshpun, S. A., and Donnelly, J. 1997. Techniques for dispersion of microorganisms into air. Aerosol Sci Technol. 27:405-421.

24. Sinha, S. 1946. Decay of certain fruits in storage. Proc. Indian Acad. Sci. 24:198-205.

25. Snowdon, A. L. 1990. A Colour Atlas of Post-harvest Diseases and Disorders of Fruit and Vegetables, Vol. 1. General Introduction and Fruit. Wolfe Scientific, London.

26. South African Department of Agriculture, Forestry and Fishery. 2011. Agricultural product standards act no. 119 of 1990; Standards and requirements regarding control of the export of citrus fruit: Amendment. Regul. Gaz. No.34233. 
27. Sugar, D. 2009. Influence of temperature and humidity in management of postharvest decay. Stewart Postharvest Rev. 5:1-5.

28. Tanaka, T. 1969. Misunderstanding with regard to citrus classification and nomenclature. Bull. Univ. Osaka Prefect. Ser. B. 21:139-145.

29. Tanaka, T. 1977. Fundamental discussion of citrus classification. Stud. Citrol. 14:1-6.

30. Van Eeden, M and Korsten, L. 2013. Alternative disease assessment method for Cercospora spot (Pseudocercospora purpurea (Cooke) Deighton) of avocado (Persea americana Mill.). Curr. Biotechnol. 2:106-113.

31. Vermeulen, H., Jordaan, D., Korsten, L., and Kirsten J. 2006. Private standards, handling and hygiene in fruit export supply chain: a preliminary evaluation of the economic impact of parallel standards. Working Pap. No. 2, Department of Agricultural Economics, University of Pretoria, Pretoria,
Gauteng, South Africa. www.researchgate.net

32. Vilanova, L., Teixidó, N., Torres, R., Usall, J., and Viñas, I. 2012. The infection capacity of $P$. expansum and $P$. digitatum on apples and histochemical analysis of host response. Int. J. Food Microbiol. 157:360-367.

33. Vilanova, L., Viñas, I., Torres, R., Usall, J., Buron-Moles, G., and Teixidó, N. 2014. Increasing maturity reduces wound response and lignification processes against Penicillium expansum (pathogen) and Penicillium digitatum (non-host pathogen) infection in apples. Postharvest. Biol. Technol 88:54-60.

34. Vilanova, L., Viñas, I., Torres, R., Usall, J., Jauset, A. M., and Teixidó, N. 2012. Infection capacities in the orange-pathogen relationship: compatible (Penicillium digitatum) and incompatible (Penicillium expansum) interactions. Food Microbiol. 29:56-66. 